\title{
Mobility management through access network discovery and selection function for load balancing and power saving in software-defined networking environment
}

Shun-Neng Yang ${ }^{1,2^{*}}$, Cheng-Hsu Ke ${ }^{2}$, Yi-Bing Lin ${ }^{2}$ and Chai-Hien Gan ${ }^{1}$

\begin{abstract}
The mobile traffic has grown rapidly with the popularity of smart mobile devices. To accommodate increasing traffic, heterogeneous network integration is considered as a viable solution. By overlapping the coverage of heterogeneous networks (e.g., the long-term evolution (LTE) and Wi-Fi integrated network), the mobile operators can use the offloading service (e.g., Wi-Fi offloading) to reduce network congestion. In this approach, a proper network coordination mechanism is required for load balancing of the LTE and Wi-Fi integrated network. In this paper, we use access network discovery and selection function (ANDSF) to suggest selection of proper base stations (e.g., LTE evolved Node Bs or Wi-Fi access points) to user equipment (UE) for load balancing. We integrate the ANDSF with software-defined networking (SDN) to make the ANDSF-aided network more programmable, flexible, and dynamically manageable. Moreover, we propose a power-saving ANDSF (PSA) algorithm to appropriately assign network resource to UEs and reduce the power consumption of Wi-Fi access points (APs). We have implemented the SDN-based PSA and measured the delay times. We also conduct simulation experiments to show that the successful probability of UEs' resource requests to PSA is almost the same as the proposed schemes of the previous studies when the network traffic is unbalanced. Our study indicates that for unbalanced traffic, PSA can reduce $15.63 \%$ more power consumption of Wi-Fi AP than the previous approaches.
\end{abstract}

Keywords: Access network discovery and selection function (ANDSF), Power saving, Software-defined networking (SDN), Long-term evolution (LTE), Wi-Fi

\section{Introduction}

In the recent years, the mobile traffic has grown rapidly with the popularity of smart mobile devices. Besides broadband mobile applications, emerging networks like Internet of Things (IoT) [1] and wireless sensor networks (WSN) [2, 3] also generate massive traffic during operation (e.g., monitoring the environment parameters to avoid disaster crisis [4]). To accommodate increasing traffic, some mobile operators have deployed Wi-Fi access points (APs) together with the long-term evolution (LTE) evolved Node Bs (eNBs) to provide Wi-

\footnotetext{
* Correspondence: takeshi@itri.org.tw; snyang@cs.nctu.edu.tw

${ }^{1}$ Information and Communications Research Laboratories, ITRI, Chutung, Hsinchu 31040, Taiwan, Republic of China

${ }^{2}$ Department of Computer Science, National Chiao Tung University, Hsinchu 30010, Taiwan, Republic of China
}

Fi offloading service to reduce network congestion [5]. To do so, a proper network coordination mechanism is required for load balancing of the LTE and Wi-Fi integrated network.

The 3rd Generation Partnership Project (3GPP) has proposed a 3GPP and non-3GPP access integrated network (e.g., the LTE and Wi-Fi integrated network) with access network discovery and selection function (ANDSF) $[6,7]$ to support network selection. In this mechanism, when a user equipment (UE) connects to the mobile network (e.g., LTE network), the ANDSF server will provide inter-system mobility policy (ISMP) and access network discovery information for the UE to select the access technology (e.g., Wi-Fi or LTE). In addition, the UE sends its location information to the ANDSF server to update its UE location database. According to a base station list 
provided by the ANDSF server, the UE can select an appropriate base station for better network traffic distribution. In this paper, we implement the ANDSF mechanism for the LTE and Wi-Fi integrated network in the softwaredefined networking (SDN) environment [8].

SDN separates the control plane and the data plane of legacy switches so that the network nodes (e.g., SDN switches) are only responsible for data forwarding, which makes the network management more simple and convenient. The SDN controller utilizes OpenFlow [9] to interact with the SDN switches, and they are called the OpenFlow Controller (OFC; e.g., OpenDaylight [10]) and the OpenFlow Switches (OFSs), respectively. SDN network management introduces a flow control application that maintains the routing rules of the OFSs through the OFC. In this way, SDN makes the network more programmable, flexible, and dynamically manageable. Specifically, the SDN can dynamically adjust the data routing path to avoid network congestion.

OpenDaylight utilizes Open Services Gateway initiative (OSGi) framework to provide network management functions [11]. OSGi framework is a modular system for Java [12]. With OSGi, OpenDaylight can dynamically add or remove a function module while it is running. The function module is called a bundle in OSGi framework [13]. In this paper, the ANDSF server is implemented at the OpenDaylight bundle level, which configures the OFSs to establish appropriate data routing paths. In this way, the ANDSF messages can be directly delivered to the ANDSF server by OFPT_PACKET_IN messages [9] when they arrive at the OFSs.

In the radio access network, we combine ANDSF with the workload-awareness base station technology to achieve load balance. With the workload-awareness feature, the mobile core network and Wi-Fi APs can update a base station's workload database. The ANDSF server then queries the database and determines whether the network traffic is required to adjust. If so, the ANDSF server suggests a list of base stations (e.g., light-loaded base stations) to the UE. The UE selects a base station in the list with its local policy (e.g., user preference settings and access history). If the UE decides to switch the connection to a light-loaded base station, the ANDSF server can help to modify the data path through the OFPT_FLOW_MOD message of OpenFlow [9].

Figure 1 depicts a traditional LTE and Wi-Fi integrated network architecture where the ANDSF server (Fig. 1 (5)) connects to the packet data network gateway (P-GW; see Fig. 1 (4)) in the mobile core network [6] through a legacy switch (Fig. 1 (6)). An ANDSF client is installed in the UE (Fig. 1 (1)) to interact with the ANDSF server. The UE connects to the external data network through either a LTE eNB (Fig. 1 (2)) or a Wi-Fi AP (Fig. 1 (3)) in the radio access networks. Thus, the UE can communicate with the ANDSF server and the application server (Fig. 1 (7)) in the external data network through the LTE or the Wi-Fi network. The Domain Name System server (DNS server; see Fig. $1(8)$ ) is responsible for providing DNS service to the UE.

In this paper, the radio access networks and the external data network are connected through SDN to provide more flexible data delivery (e.g., through the OFPT_FLOW_MOD message, we can switch the data delivery path to a light-loaded base station to achieve load balance). The details of the proposed network architecture and the implementation are presented in Section 2. Section 3 describes two SDN-based ANDSFaided inter-system change procedures and measures the delay times of these procedures. Section 4 proposes a power-saving ANDSF algorithm and the performance evaluation for the ANDSF server bundle (ASB). Conclusions are given in Section 5 .

\section{ANDSF in the SDN environment}

This section describes the multi-radio access technology (multi-RAT), the SDN-based network architecture, and our implementation.

Figure 2 depicts the SDN-based network with the ANDSF server bundle (ASB). The UE (Fig. 2 (1)) connects to the application server (Fig. 2 (8)) in the external data network through the radio access networks. The radio access networks consist of LTE eNBs (Fig. 2 (2)) and Wi-Fi APs (Fig. 2 (3)). The LTE eNB connects to an OpenFlow Switch (OFS; see Fig. 2 (7)) through the P-GW (Fig. 2 (4))

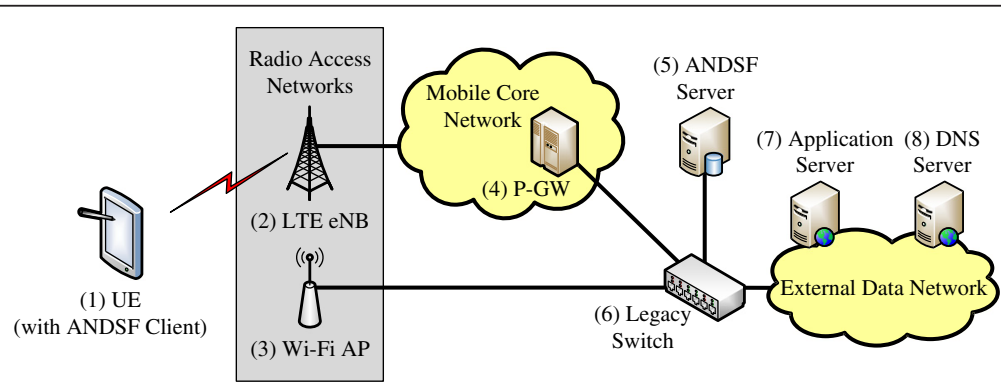

Fig. 1 Traditional network with ANDSF 


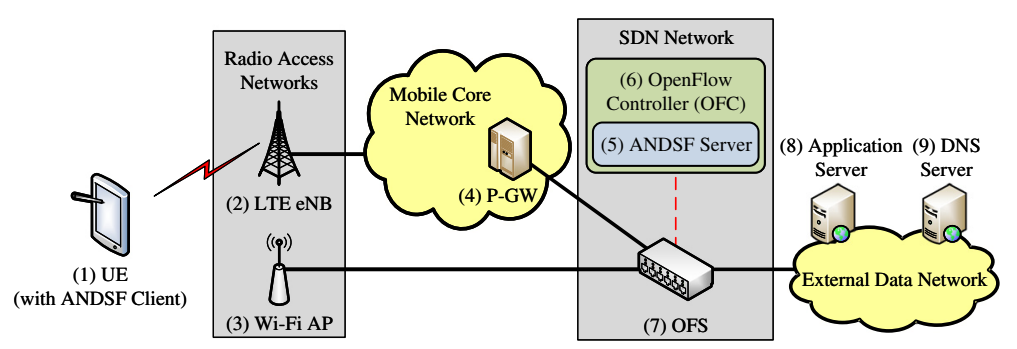

Fig. 2 SDN-based network with ASB

in the mobile core network while the Wi-Fi AP directly connects to the OFS. The OFS is controlled by the OpenFlow Controller (OFC; see Fig. 2 (6)). The ANDSF server bundle (ASB; see Fig. 2 (5)) is installed in the OFC and responsible for processing the ANDSF related messages from the ANDSF client of the UE. In this paper, the ASB is also responsible for creating flow entries for packet routing on the OFS. The DNS server (Fig. 2 (9)) provides DNS service to the UE.

The ASB maintains a database for storing information related to base stations (e.g., LTE eNBs and Wi-Fi APs) and UEs. The information includes base station's basic service set identifier (BSSID), service set identifier (SSID) and workload, and UE's location. The ASB communicates with the ANDSF client by the ANDSF protocol [6, 7]. The server also provides a list of base stations (e.g., Wi-Fi APs) to be selected by the UE.

We implement an experimental environment according to the network architectures in Figs. 1 and 2. In the experimental environment, we use the Huawei BBU3900 TD-LTE eNB [14] as the LTE eNB ((2), Figs. 1 and 2), and the D-Link DIR-835 Wi-Fi AP is used as the Wi-Fi AP ((3), Figs. 1 and 2) in the radio access networks. The ng4T NG40 Evolved Packet Core (EPC) emulator serves as the mobile core network ((4), Figs. 1 and 2). We use OpenDaylight controller and HP 3800 series switch (in SDN-enable mode) as the OFC (Fig. 2 (6)) and OFS (Fig. 2 (7)) in the SDN network. In the traditional network architecture, we use the HP 3800 series switch in SDN-disable mode as the legacy switch (Fig. 1 (6)).

We propose two methods for the OFC to modify the routing rules on the OFSs to set up a new data path when the UE moves from one radio access network (e.g., LTE) to another (e.g., Wi-Fi). The first method sets the new routing rule through the table-miss event (i.e., a data packet does not match any flow entry in the flow table of an OFS) defined in the OpenFlow protocol [9]. The second method uses the original connection of the UE to notify the ASB its target base station, and then the ASB modifies the routing rules on the OFS to speed up the process of directing the packets to the target base station. Details of the proposed methods are described in Section 3.

\section{ANDSF-aided inter-system change procedure}

This section presents two ANDSF-aided inter-system change procedures. Switching the UE's radio access between different radio access technologies is called intersystem change [15]. In this section, we first describe the sequential inter-system change procedure with the ANDSF protocol $[6,7]$. In this procedure, the routing rule establishment of the target network (e.g., Wi-Fi network) is triggered by the table-miss event [9] of the SDN network. The table-miss event occurs after the UE connects to the Wi-Fi AP and starts to send data. To speed up this process, we introduce the parallel inter-system change procedure. In the parallel alternative, the routing rules modification process is executed in parallel with the UE's authentication process at the target network. We also measure the delay times of the proposed procedures in the end of this section.

\subsection{The sequential inter-system change procedure}

Figure 3 illustrates the sequential inter-system change procedure in SDN with the following steps:

Step 1. The UE establishes the network access to LTE.

Step 2. The data packets are sent to the OFS through the LTE eNB.

Step 3. The first arriving packet triggers a table-miss event in the OFS [9]. The OFS then forwards the packet encapsulated in the OFPT_PACKET_IN message to the ASB in the OFC.

Step 4. The ASB analyzes the packet by checking the source IP and the destination IP, calculates the routing path and generates the corresponding flow entries.

Step 5. The ASB adds the flow entries in the OFS and sends the OFPT_FLOW_MOD message to the OFS. The flow entries are set as follows: If the destination IP of a packet is IP_Application_Server (i.e., the IP address of the application server), the OFS forwards this packet to the application server. If the destination IP of a packet is IP_LTE (i.e., the IP address of the UE's LTE interface), the OFS forwards it to the LTE eNB. 


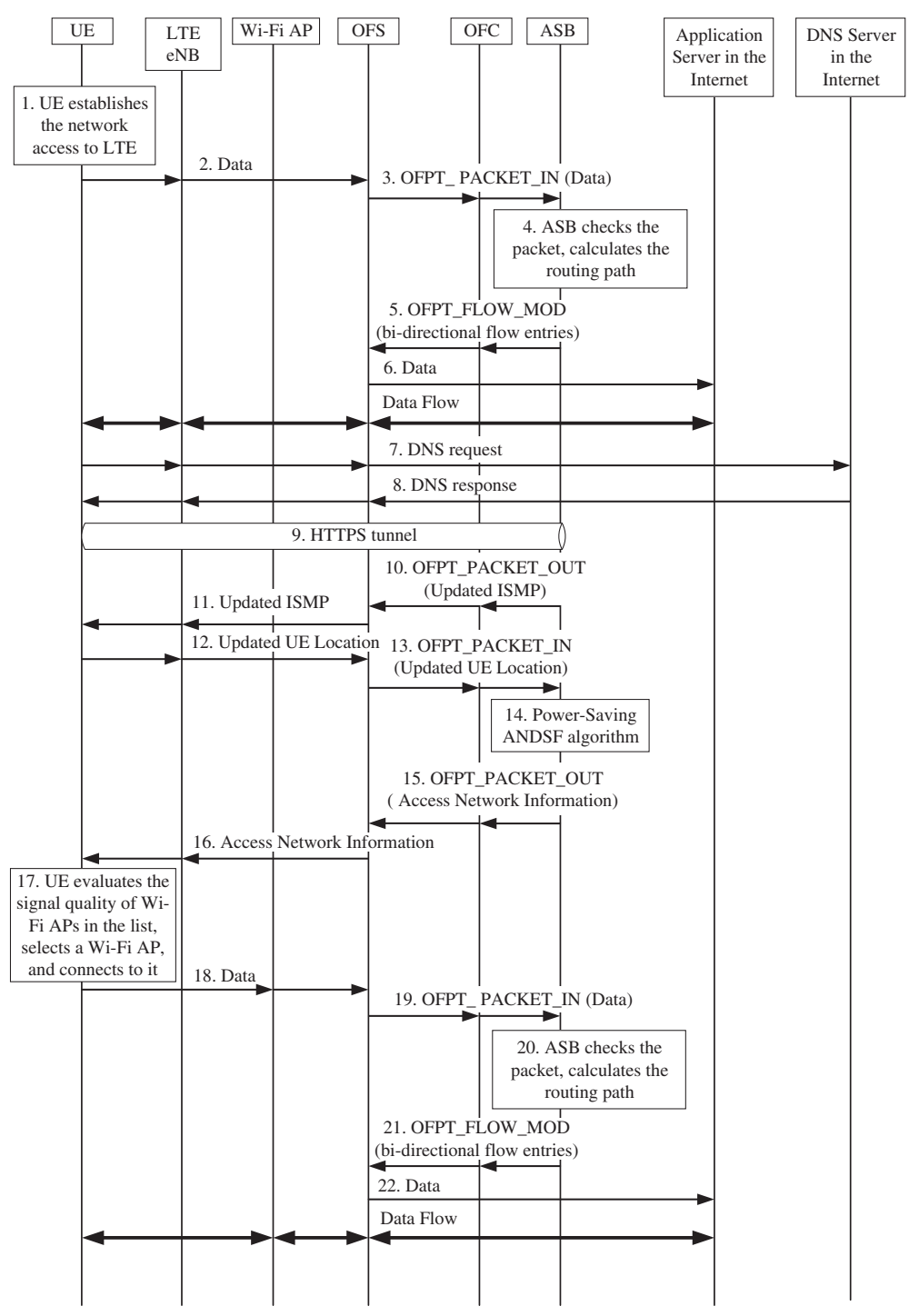

Fig. 3 Sequential inter-system change procedure

Step 6. The packet matches the flow entry "the destination IP is IP_Application_Server" in the OFS and is sent to the application server.

Step 7. The UE discovers the ASB by using domain name system (DNS) lookup [7]. The UE sends a DNS request to the DNS server through the LTE eNB. The request includes the fully qualified domain name (FQDN) of ANDSF service name (ANDSF-SN) [16]. The data path setting between the UE and the DNS server is similar to steps 2-6, and the details are omitted.

Step 8. The DNS server replies a DNS response to the UE. The response contains the IP address of the ASB (i.e., ANDSF_IP).

Step 9. The UE establishes an HTTPS tunnel to the ASB. The UE and the ASB authenticate each other based on shared key $[17,18]$. The shared key shall be used as a master key to generate Transport Layer Security (TLS) session keys.

Step 10.The ASB sends an updated inter-system mobility policy (ISMP) message encapsulated in the OFPT_PACKET_OUT message to the OFS.

Step 11.The OFS receives the OFPT_PACKET_OUT message, retrieves the updated ISMP message and forwards it to the UE.

Step 12.The UE sends an updated UE Location message to the OFS through the LTE eNB.

Step 13.The destination IP of the UE Location message packet is configured as $A N D S F_{-} I P$, which matches the preset flow entry "the destination 
IP is ANDSF_IP”. The OFS sends the packet to the OFC through the OFPT_PACKET_IN message. The OFC forwards this message packet to the ASB.

Step 14. The ASB calculates the current location of the UE and selects proper base stations (e.g., the neighboring Wi-Fi APs of the UE) according to a power-saving ANDSF algorithm (the details are described in Section 4).

Step 15. The ASB sends an access network information message encapsulated in the OFPT_PACKET_OUT message to the OFS. The information includes the list of available base stations' IDs (e.g., the SSIDs of Wi-Fi APs) of the access network and the corresponding public land mobile network (PLMN) IDs of these base stations.

Step 16. The OFS receives the OFPT_PACKET_OUT message, retrieves the Access Network Information message, and forwards it to the UE.

Step 17.Upon receipt of the Access Network Information message, the UE evaluates the radio signal quality of the base stations (e.g., Wi-Fi APs) in the available base station list. The UE then selects a Wi-Fi AP in the list according to its local policy (e.g., the Wi-Fi AP whose signal strength is higher than a signal strength threshold $T_{\mathrm{s}}$ ) and connects to it.

Steps 18-22. These steps illustrate how the UE sends the data packets to the application server through the Wi-Fi AP, which are similar to steps 2-6, and the details are omitted.

\subsection{The parallel inter-system change procedure}

Figure 4 illustrates the parallel inter-system change procedure. In this procedure, the UE notifies the ASB of the selected base station ID in parallel with the UE connects to the selected base station. Upon receipt of the selected base station's information, the ASB modifies the routing rule while the UE authenticates and connects to the new base station. The procedure is described in the following steps:

Steps 1-16. Same as steps 1-16 in Section 3.1.

Step 17. The UE receives the Access Network Information message, and evaluates the radio signal quality of the base stations (e.g., Wi-Fi APs) in the available base station list. The UE then selects a Wi-Fi AP in the list according to its local policy (e.g., the Wi-Fi AP whose signal strength is higher than the signal strength threshold $T_{\mathrm{s}}$ ).

Step 18. The UE sends the information message of the selected Wi-Fi AP (e.g., Wi-Fi AP's SSID) to the OFS through the LTE eNB.

Step 19. The destination IP of the selected Wi-Fi AP information message packet is configured as ANDSF_IP, which matches the preset flow entry in the OFS (same as that in step 13 of Section 3.1). Therefore, the OFS forwards the message through the OFPT_PACKET_IN message to the ASB in the OFC.

Step 20.The ASB analyzes the packet by checking the source IP and the information message of the selected Wi-Fi AP, calculates the routing path and generates the corresponding flow entries.

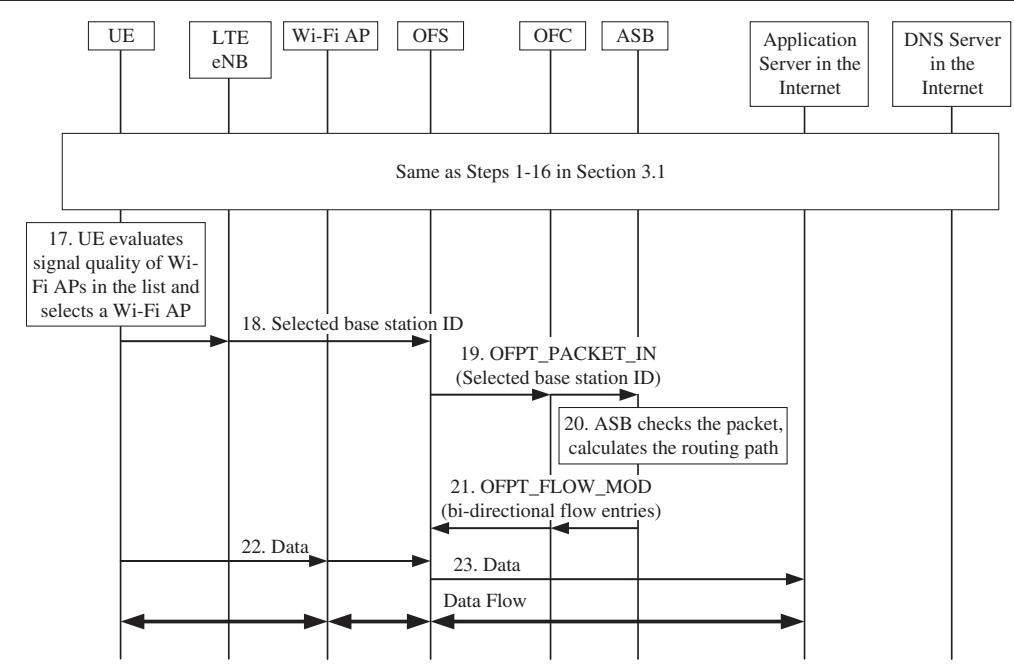

Fig. 4 Parallel inter-system change procedure 
Step 21. The ASB adds a new flow entry to the OFS through the OFPT_FLOW_MOD message. The flow entry is "if the destination IP is $I P_{-} W i-F i$ (i.e., the IP address of UE's Wi-Fi interface), then the packet is sent to the Wi-Fi AP."

Step 22.In parallel with step 18, the UE connects to the selected Wi-Fi AP and sends data packets to the OFS though the Wi-Fi AP.

Step 23. The data packets are delivered to the application server through the OFS.

Different from the sequential procedure in Section 3.1, the UE sends the selected Wi-Fi AP information to the ASB through the LTE eNB (steps 18-19) and connects to this Wi-Fi AP (step 22) in parallel. The flow entry could be set up before the UE starts to send data through the Wi-Fi AP, and thus, this mechanism reduces the time complexity of the inter-system change procedure. We measure the delay times of the sequential and parallel inter-system change procedures and compare them with the delay time of the traditional (non-SDN) network environment illustrated in Fig. 1. In the experimental environment mentioned in Section 2, we assume the connection and authentication process time of the Wi-Fi network is $380 \mathrm{~ms}$ according to [19]. The results show that the delay time for the non-SDN procedure is $524 \mathrm{~ms}$, the sequential procedure saves $18 \mathrm{~ms}(3.44 \%)$, and the parallel one can save $25 \mathrm{~ms}(4.77 \%)$. We also measure the delay times of above procedures with loaded traffic. We generate the loaded traffic by two opposite direction data traffic (each has $1000 \mathrm{Mb} / \mathrm{s}$ data rate) on the HP 3800 series switch. The delay times of the non-SDN, sequential, and parallel procedures with loaded traffic are 531, 524, and $502 \mathrm{~ms}$, respectively. If the connection and authentication process time can be reduced to $90 \mathrm{~ms}$ [19], the delay time for the non-SDN procedure will be $221 \mathrm{~ms}$, the sequential and the parallel ones can save $6 \mathrm{~ms}(2.71 \%)$ and $13 \mathrm{~ms}(5.88 \%)$, respectively. Moreover, the delay times of the non-SDN, sequential, and parallel procedures with loaded traffic will become 234, 236, and $226 \mathrm{~ms}$ respectively. Except for the connection and authentication process time, the rest part of the delay time includes the Wi-Fi network interface configuration time of the operating system and the routing rule setup time of the Wi-Fi AP.

\section{Power-saving ANDSF algorithm and performance evaluation}

Before the ASB suggests a list of base stations to a UE, it must confirm that these base stations have enough radio resources (i.e., bandwidth) to accommodate this UE. We assume that each base station has bandwidth of $B \mathrm{Mb} / \mathrm{s}$. When a resource attempt (i.e., a UE tries to access network through a base station or hand over to a base station) arrives, the base station allocates a fixed bandwidth $b \mathrm{Mb} / \mathrm{s}$ to the UE. This process is called resource assignment. In this way, a base station can serve $B / b$ UEs.

We propose a power-saving ANDSF (PSA) algorithm that selects proper base stations for the UE with load balance and power saving. Originally, ANDSF was designed for load balance, which is an important issue when the network is congested. On the other hand, when the workload of the network is light, many base stations do not serve any UE. We mark these base stations as "inactive." The inactive base stations consume power without providing any service. Thus the idea is to assign UEs to active APs, and keep inactive APs inactive. Then we perform load balancing in the selection of active APs. When a location is covered by more than one base station, the PSA algorithm attempts to turn off some inactive APs. The APs are turned on when the number of resource attempts increases. The PSA algorithm combines both repacking technique and workload information to achieve power saving and load balance. Some studies have focused on resource assignment in the traditional networks. Two schemes called no repacking (NR) and repacking on demand (RoD) were proposed [20]. Suppose that a UE requests for network resource. The NR scheme first tries to allocate resource of the $\mathrm{Wi}-\mathrm{Fi} \mathrm{AP}$ to the UE. If the Wi-Fi AP is not available, the resource attempt of the UE is transferred to the LTE eNB. If the LTE eNB cannot accommodate this request, the request is rejected. To improve the performance of NR, repacking techniques reduce the probability of rejecting resource attempts as follows. Consider a Wi-Fi AP that does not have enough resource to accommodate the request of a UE. In NR, this UE will be served by the LTE eNB. If Wi-Fi network resource is available later, then through the repacking technique, this UE can be transferred to the $\mathrm{Wi}-\mathrm{Fi}$ again. The process of switching a UE from the LTE eNB to the Wi-Fi AP is called repacking. Repacking increases the network resource in the LTE eNB so that more LTE eNB resource can be shared by the UEs when Wi-Fi AP resource is not available to these UEs. In $\mathrm{RoD}$, repacking is exercised when a user equipment UE1 cannot access any resource in the radio coverage of both the Wi-Fi AP and the LTE eNB. In this case, RoD is exercised to identify repacking candidates. Every repacking candidate is a user equipment UE2 that satisfies the following criteria:

Criterion 1. UE2 occupies the resource in the LTE eNB. Criterion 2. The Wi-Fi AP that covers UE2 has available resource to serve UE2.

RoD selects one of the repacking candidates to hand over from the LTE eNB to the Wi-Fi AP, and then the 
LTE eNB can reclaim the resource to serve UE1. If RoD cannot find any repacking candidate, the resource attempt of UE1 is rejected.

\subsection{Power-saving ANDSF algorithm}

In the power-saving ANDSF (PSA) algorithm, we turn off a base station when no UE is using the resource in this base station. When a UE releases the resource from a Wi-Fi AP, we check if it is appropriate to turn off the Wi-Fi AP. If so, we move all UEs on the Wi-Fi AP to its neighboring base stations and turn it off. In this way, we can achieve power saving. When a resource attempt arrives, the PSA algorithm takes workload information of all active base stations into consideration, and suggests the UE to use one of the active Wi-Fi APs with the lowest workload. If no resource is available in the Wi-Fi AP, the resource attempt of the UE is transferred to the LTE eNB. In this way, we can achieve load balance. If the UE cannot find any resource in both active Wi-Fi APs and LTE eNB, we first execute the repacking process. If the UE still cannot acquire the resource, we try to turn on an appropriate Wi-Fi AP to provide the network resource. If we cannot turn on any Wi-Fi AP to accommodate this UE, then we try to turn on other Wi-Fi AP under the same coverage of LTE eNB to execute repacking process. The details of the PSA algorithm are shown in Fig. 5 with the following steps:

Step 1. Check if any active Wi-Fi AP can accommodate the UE's resource attempt. If so, go to step 2. Otherwise, go to step 3 .

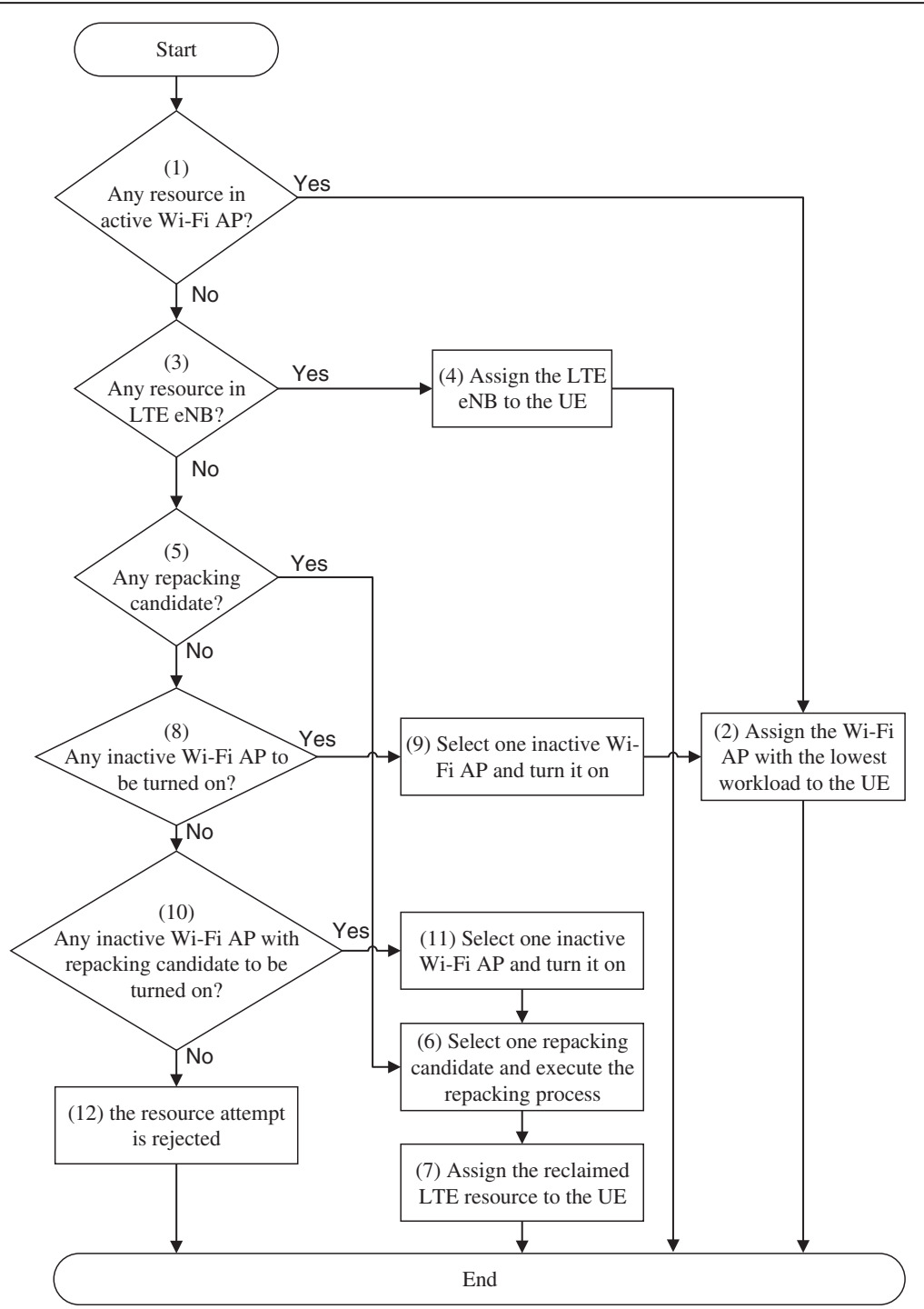

Fig. 5 Flow chart for the PSA algorithm 
Step 2. Assign the Wi-Fi AP with the lowest workload to the UE and the algorithm is terminated.

Step 3. Check if the LTE eNB's resource is available for the attempt. If so, go to step 4. Otherwise, go to step 5.

Step 4. Assign the LTE eNB to the UE and the algorithm is terminated.

Step 5. Check if there is any repacking candidate. If so, go to step 6. Otherwise, go to step 8.

Step 6. Randomly select one repacking candidate and execute the repacking process. Go to step 7 .

Step 7. Assign the reclaimed LTE resource to the UE and the algorithm is terminated.

Step 8. Check if there is any inactive Wi-Fi AP that can be turned on to accommodate this resource attempt. If so, go to step 9. Otherwise, go to step 10.

Step 9. Select one of the inactive Wi-Fi APs and turn it on. Go to step 2.

Step 10.Check if there is any inactive Wi-Fi AP with repacking candidate. If so, go to step 11. Otherwise, go to step 12 .

Step 11.Select one of the inactive Wi-Fi APs and turn it on. Go to step 6.

Step 12.Reject the resource attempt and the algorithm is terminated.

\subsection{System model for simulation}

This section describes the input parameters and output measures for the resource assignment model. We consider a wrapped mesh heterogeneous network with uniform request traffic as shown in Fig. 6 (and will consider hotspot traffic in Fig. 8). This configuration includes four LTE eNBs and 64 Wi-Fi APs. Each LTE eNB covers $4 \times 4$ Wi-Fi APs. The boundary base station effect can be ignored by using the wrapped topology [21]. When a UE tries to access the network, the UE connects to one of the $3 \times 3$ neighboring Wi-Fi APs or the LTE eNB that covers its location. After obtaining the radio and the core network resources, the UE is connected for a holding time $T_{\mathrm{H}}$, and then releases the resource. We assume that the UE stays at the Wi-Fi AP's coverage with the residence time $T_{\mathrm{R}}$. If $T_{\mathrm{H}} \geq T_{\mathrm{R}}$, then the UE moves to one of the four neighboring Wi-Fi APs (i.e., coverage of the upward, downward, leftward, and rightward APs) with the same probability (i.e., 0.25 ).

Three types of input parameters are considered in our model, including the system, traffic, and mobility parameters.

System parameters: Every LTE eNB and every Wi-Fi AP support bandwidths of $B_{\mathrm{L}}$ and $B_{\mathrm{W}} \mathrm{Mb} / \mathrm{s}$, respectively. The base station allocates bandwidth $b \mathrm{Mb} / \mathrm{s}$ to

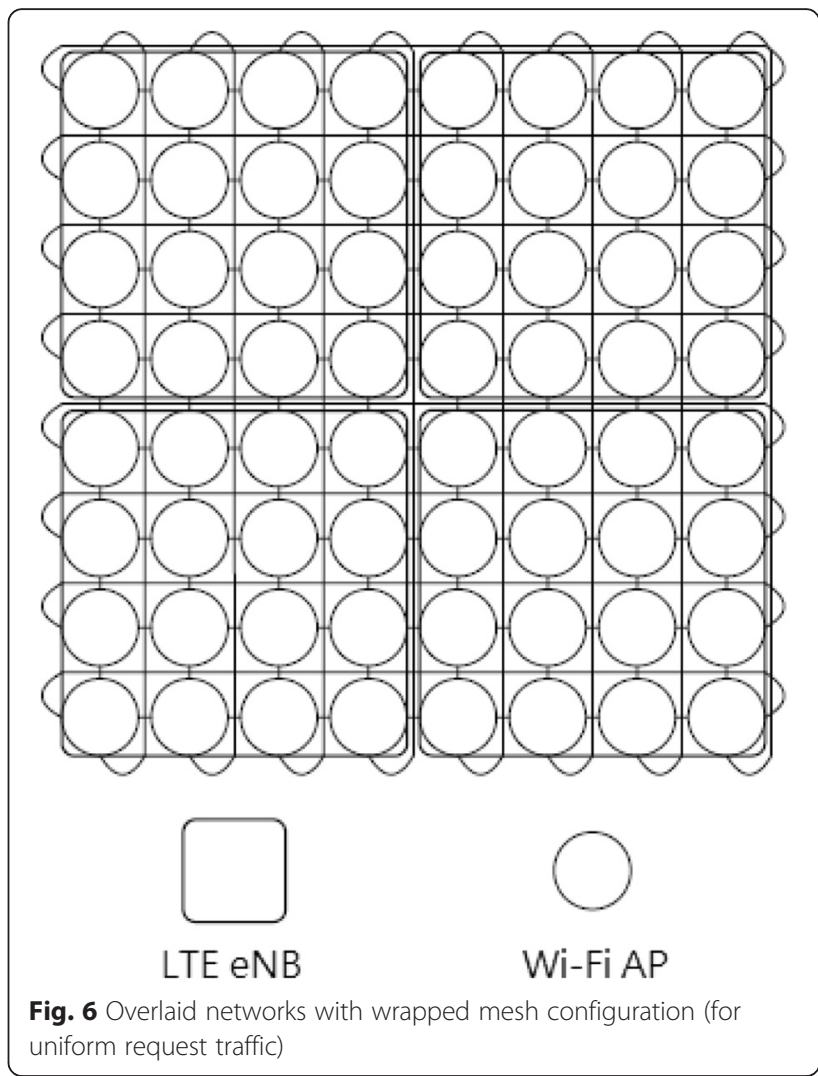

each resource attempt, so that the LTE eNB and the Wi-Fi AP can serve $N_{\mathrm{L}}$ (i.e., $B_{\mathrm{L}} / b$ ) and $N_{\mathrm{W}}$ (i.e., $B_{\mathrm{W}} / b$ ) UEs, respectively.

Traffic parameters: The resource attempt arrivals are a Poisson process [22] with the rate $\lambda$. The resource holding time $T_{\mathrm{H}}$ has a gamma distribution with the mean $1 / \mu$ and the variance $V_{\mu}$.

The residence time $T_{R}$ of a UE at the radio coverage of a Wi-Fi AP has a gamma distribution with the mean $1 / \eta$ and the variance $V_{\eta}$.

The output measures of our model are defined as follows:

$P_{\mathrm{s}}$ : the probability that a UE's resource attempt is successful

$P_{\mathrm{f}}$ : the probability that a UE's resource attempt is rejected; i.e., $P_{\mathrm{f}}=1-P_{\mathrm{s}}$

$M_{\mathrm{AP}}$ the mean number of active Wi-Fi APs

Note that the less the $M_{\mathrm{AB}}$ the more the power is saved.

\subsection{Results and discussion}

Based on the system model described in Section 4.2, we compare NR, RoD, and PSA in terms of output measures. We use the discrete-event-driven scheme [23] to simulate the arrival, resource holding, and mobility 

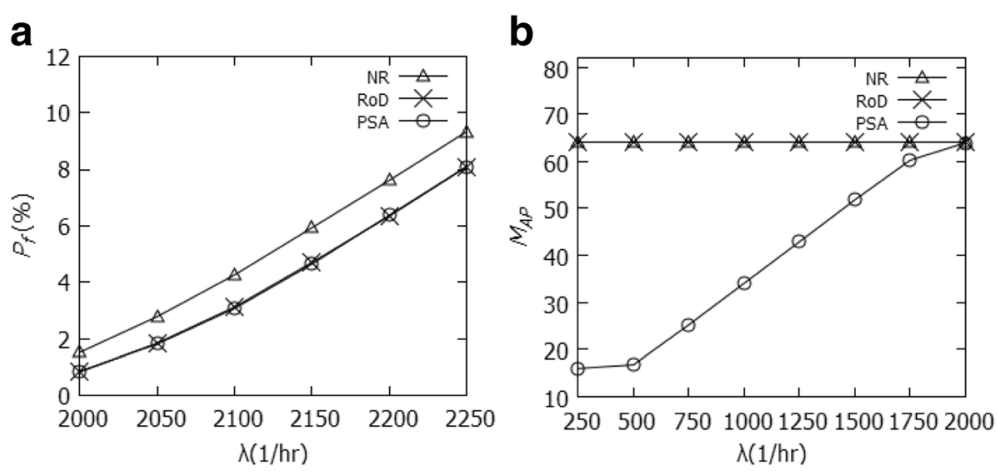

Fig. 7 Effects of $\lambda$ on $P_{f}$ and $M_{A P}\left(N_{L}=8, N_{W}=10,1 / \mu=0.005(h), V_{\mu}=1 / \mu^{2}, 1 / \eta=0.5(h)\right.$, and $\left.V_{\eta}=1 / \eta^{2}\right)$. a Effects of $\lambda$ on $P_{f}$. b Effects of $\lambda$ on $M_{A P}$

behavior of the UEs in the simulation model. We simulate $1,000,000$ resource attempts in each simulation.

Figure 7a plots $P_{\mathrm{f}}$ as an increasing function of $\lambda$ from 2000 to $2250(1 / \mathrm{h})$ where $N_{\mathrm{L}}=8, N_{\mathrm{W}}=10,1 / \mu=0.005$ (h), $V_{\mu}=1 / \mu^{2}, 1 / \eta=0.5$ (h), and $V_{\eta}=1 / \eta^{2}$. The above parameters are set up according to [20]. It shows that PSA can support almost the same number of resource attempts as RoD in Fig. 7a. Also, PSA can support more resource attempts than NR.

Figure 7b plots the $M_{\mathrm{AP}}$ curve of PSA, which is an increasing function of $\lambda$ from 250 to $2000(1 / \mathrm{h})$ where $N_{\mathrm{L}}=8, N_{\mathrm{W}}=10,1 / \mu=0.005(\mathrm{~h}), V_{\mu}=1 / \mu^{2}, 1 / \eta=0.5(\mathrm{~h})$, and $V_{\eta}=1 / \eta^{2}$. The $M_{\mathrm{AP}}$ values of NR and RoD in Fig. $7 \mathrm{~b}$ remain the same. On the other hand, PSA saves more power than NR and RoD (i.e., the PSA keeps more Wi-Fi APs inactive and thus saves more power of APs) when $\lambda$ is lower than $2000(1 / \mathrm{h})$.

We then consider the hotspot request traffic as shown in Fig. 8. Except for the hotspot area (i.e., the area which has the request arrival rate higher than 5000), other Wi-Fi APs only cover few users (50 or $5 \%$ of the hotspot area).
Table 1 shows the performance of NR, RoD, and PSA with the hotspot traffic in Fig. 8. The table indicates that PSA can support $99.8 \%$ resource attempts of RoD. On the other hand, as compared to NR and RoD, PSA can reduce $(64-54) / 64 \fallingdotseq 15.63 \%$ of the AP power consumption.

\section{Conclusions}

This paper implemented an access network discovery and selection function (ANDSF) mechanism. Unlike the ANDSF solution, we proposed a power-saving ANDSF (PSA) algorithm in the SDN network environment. Through this mechanism, a UE only needs to install the ANDSF client software to communicate with the ANDSF server bundle (ASB). The ASB is an ANDSF application in the SDN controller, which can achieve load balance and power saving by exercising the PSA algorithm. We measured the delay times in the prototype we developed. We also conducted simulation experiments to investigate the performance of the PSA algorithm on the success probability $P_{\mathrm{s}}$ and the mean number of active Wi-Fi APs $M_{\mathrm{AP}}$. The simulation

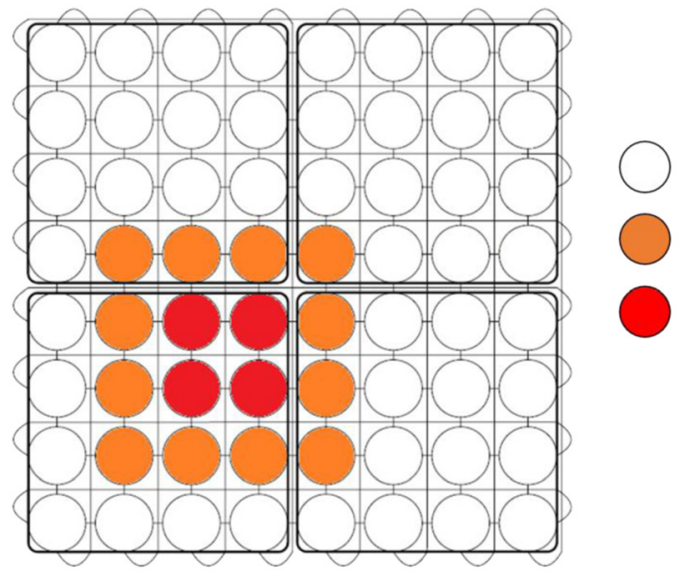

Arrival rate: 500 per hr

Arrival rate: 5000 per hr

Arrival rate: 10000 per hr

Fig. 8 Overlaid networks with wrapped mesh configuration (for hotspot request traffic) 
Table 1 Performance of NR, RoD, and PSA with the hotspot traffic

\begin{tabular}{llll}
\hline & NR & RoD & PSA \\
\hline$P_{\mathrm{s}}$ & $91.72 \%$ & $92.69 \%$ & $92.5 \%$ \\
$M_{\text {AP }}$ & 64 & 64 & 54 \\
\hline
\end{tabular}

results show that $P_{\mathrm{s}}$ of PSA is $92.5 \%$, which is $99.8 \%$ that of repacking on demand (RoD). Compared to no repacking (NR) and $\mathrm{RoD}$, PSA reduces $15.63 \%$ of the AP power consumption. To conclude, PSA can effectively achieve load balance as well as power saving.

\section{Acknowledgements}

This work was supported by Academia Sinica AS-102-TP-A06, the ICL/ITRI Project, Microsoft 103Q733, D-link 103C142, MOST 104-2218-E-009-009, and the MoE ATU plan.

\section{Competing interests}

The authors declare that they have no competing interests.

Received: 22 April 2016 Accepted: 26 August 2016

Published online: 02 September 2016

\section{References}

1. T Robles, R Alcarria, D Martín, M Navarro, R Calero, S Iglesias, M López, An IOT based reference architecture for smart water management processes. Journal of Wireless Mobile Networks, Ubiquitous Computing, and Dependable Applications (JoWUA) 6(1), 4-23 (2015)

2. DG Jiménez, FL Gall, Testing a commercial sensor platform for wideband applications based on the 802.15.4 standard. Journal of Wireless Mobile Networks, Ubiquitous Computing, and Dependable Applications (JoWUA) 6(1), 24-36 (2015)

3. F-Y Leu, H-L Chen, C-C Cheng, Improving multi-path congestion control for event-driven wireless sensor networks by using TDMA. Journal of Internet Services and Information Security (JISIS) 5(4), 1-19 (2015)

4. K Takahagi, T Ishida, A Sakuraba, K Sugita, N Uchida, Y Shibata, Construction of a mega disaster crisis management system. Journal of Internet Services and Information Security (JISIS) 5(4), 20-40 (2015)

5. R Li, Aptilo and ELTA Deliver Wi-Fi Offloading to Chunghwa Telecom and FarEasTone, 2013. http://www.aptilo.com/announcements/2013/aptilo-eltawifi-offloading-to-chunghwa-telecom-and-fareastone. Accessed 25 Feb 2016

6. 3GPP TS 23.402, "Architecture Enhancement for Non-3GPP Access," v13.4.0, Dec 2015.

7. 3GPP TS 24.302, "Access to the 3GPP Evolved Packet Core (EPC) via Non3GPP Access Networks," Stage 3, v13.4.0, Dec 2015.

8. Open Networking Foundation, Software-Defined Networking, The New Norm for Networks, ONF White Paper, 2012

9. Open Networking Foundation, OpenFlow Switch Specification 1.3.4, 2014

10. Linux Foundation Collaborative Projects: OpenDaylight (2016), http://www. opendaylight.org/. Accessed 25 Feb 2016.

11. SDN Hub, OpenDaylight Application Developer's tutorial, 2015. http://sdnhub. org/tutorials/opendaylight/. Accessed 23 July 2016

12. OSGi Alliance (2016), https://WwW.osgi.org/. Accessed 23 July 2016.

13. Kanika, OpenDaylight \& OSGI (2016), http://sdntutorials.com/opendaylightand-osgi/. Accessed 23 July 2016.

14. I Poole, LTE FDD, TDD, TD-LTE Duplex Schemes (2016), http://www.radioelectronics.com/info/cellulartelecomms/lte-long-term-evolution/lte-fdd-tddduplex.php. Accessed 25 Feb 2016.

15. 3GPP TS 21.905, "Vocabulary for 3GPP Specifications," v13.0.0, Dec 2015.

16. 3GPP TS 23.003, "Numbering, Addressing and Identification," v13.4.0, Dec 2015.

17. 3GPP TS 33.222, "Generic Authentication Architecture (GAA); Access to Network Application Functions Using Hypertext Transfer Protocol over Transport Layer Security (HTTPS)," v12.3.0, Dec 2013.

18. 3GPP TS 33.310, "Network Domain Security (NDS); Authentication Framework (AF)," v13.0.0, Dec 2015.

19. A Roos, A Keller, AT Schwarzbacher, S Wieland, Sequential authentication concept to improve WLAN handover performance, in International Conference on Intelligent Interactive Assistance and Mobile Multimedia Computing, 2009
20. H-M Tsai, A-C Pang, Y-C Lin, Y-B Lin, Channel Assignment for Hierarchical Cellular Networks, in International Conference on Parallel Processing, 2003

21. Y-B Lin, W Mak, Eliminating the Boundary Effect of a Large-Scale Personal Communication Service Network Simulation. ACM Transactions on Modeling and Computer Simulation 4(2), 165-190 (1994)

22. C-H Ng, B-H Soong, Queueing Modelling Fundamentals (Wiley, Hoboken, 2008)

23. $\mathrm{H}-\mathrm{L}$ Fu, $\mathrm{P}$ Lin, H Yue, G-M Huang, C-P Lee, Group mobility management for large-scale machine-to-machine mobile networking. IEEE Transactions on Vehicular Technology 63(3), 1296-1305 (2014)

\section{Submit your manuscript to a SpringerOpen ${ }^{\circ}$ journal and benefit from:}

- Convenient online submission

- Rigorous peer review

- Immediate publication on acceptance

- Open access: articles freely available online

- High visibility within the field

- Retaining the copyright to your article

Submit your next manuscript at springeropen.com 